\title{
El cariotipo fundamental de Alstroemeria versicolor Ruiz et Pav. (Alstroemeriaceae)
}

\section{The fundamental karyotype of Alstroemeria versicolor Ruiz et Pav. (Alstroemeriaceae)}

\author{
Carlos M. Baeza ${ }^{1}$, Jaime Espejo² \& Eduardo Ruiz ${ }^{1}$ \\ 1'Departamento de Botánica, Universidad de Concepción, Casilla 160-C, Concepción, Chile. \\ ${ }^{2}$ Programa de Postgrado, Facultad de Ciencias Forestales, Universidad de Concepción, Victoria 631, Casilla 160-C, \\ Concepción, Chile. \\ cbaeza@udec.cl
}

\begin{abstract}
The karyotype of Alstroemeria versicolor Ruiz et Pav. from Chile was described. The species has a karyotype $2 \mathrm{n}=2 \mathrm{x}=16$, and the haploid formula was $1 \mathrm{~m}+3 \mathrm{sm}+1 \mathrm{st}+2 \mathrm{st}$-sat $+1 \mathrm{t}$. The reported karyotype is asymmetric (AsK \% $=65.9$ and Syi $=51.8 \%$ ). The results are discussed including data of others species.
\end{abstract}

Alstroemeria L. (Alstroemeriaceae) es un género de Liliopsida (monocotiledóneas) de América, constituido por ca. 50 especies, de las cuales la gran mayoría crece en Chile y Brasil (Bayer 1987, Muñoz \& Moreira 2003, Baeza et al. 2010 b). En Chile están citados 49 taxones, de los cuales 25 son endémicos (Muñoz \& Moreira 2003). Este género crece principalmente en la zona mediterránea de Chile, la cual presenta un alto nivel de endemismo (Arroyo 1995). En esta área crece Alstroemeria versicolor Ruiz et Pav., especie endémica que habita desde Pirque, Región Metropolitana (333's) hasta el fundo Los Alpes en la Región de la Araucanía (374's), a orillas de ríos y quebradas de cerros. Las flores de esta especie se caracterizan por presentar todos los tépalos recurvados y todos ellos con rayitas oscuras, característica no muy común dentro del género (Muñoz \& Moreira 2003). Es una de las especies chilenas que presenta una amplia distribución en el país cubriendo cuatro regiones. Todas las especies de Alstroemeria presentan un alto valor ornamental, por lo tanto el estudio de su cariotipo resulta fundamental para futuros planes de cultivo y eventual manejo.

En esta comunicación se documenta el cariotipo de $A$. versicolor y se compara con el cariotipo de otras especies de Alstroemeria de Chile que presentan baja longitud total de cromosomas (LTC).

Se estudió una población constituida por alrededor de 30 ejemplares de Alstroemeria versicolor proveniente de Chile, IX Región, Provincia de Angol, 5,9 km desde la plaza de Angol hacia la ruta 180, camino a Renaico, $80 \mathrm{~m}\left(37^{\circ} 48^{\prime} \mathrm{S} /\right.$ 7242’W), 31-XII-2009. J. Espejo s.n. Los ejemplares coleccionados están depositados en el Herbario CONC de la Universidad de Concepción, Chile.

Tanto el estudio de los cromosomas como sus mediciones y construcción del cariotipo se efectuaron siguiendo la metodología propuesta por Baeza et al. (2008, 2010a). Los tejidos meristemáticos apicales de raíces se obtuvieron de plantas cultivadas en invernadero. Para la población analizada (5 individuos, 20 placas metafásicas) se determinó el índice de asimetría del cariotipo (AsK \%) definido por Arano y Saito (1980), el índice Syi (Venora et al. 2002) y la longitud total diploide de los cromosomas (LTC en $\mu \mathrm{m}$ ). Los cromosomas se clasificaron de acuerdo a Levan et al. (1964). Para la comparación de especies de Alstroemeria con bajo LTC los datos se obtuvieron de Baeza et al. (2010 a) y Jara-Seguel et al. (2004).

Alstroemeria versicolor presenta un cariotipo asimétrico $2 \mathrm{n}=2 \mathrm{x}=16$ y una fórmula haploide $1 \mathrm{~m}+3 \mathrm{sm}+1 \mathrm{st}+$ 2 st-sat $+1 \mathrm{t}$, esto es, 1 par de cromosomas metacéntricos (par 1), 3 pares submetacéntricos (pares 2, 7 y 8), 1 par subtelocéntrico (par 6), 2 pares subtelocéntricos con satélite (pares 3 y 5) y un par (4) telocéntrico (Fig. 1). El índice de asimetría del cariotipo de Arano y Saito (AsK \%) fue de 65,9, el Syi de 51,80\% y el LTC de 129,0 $\mu \mathrm{m}$ (Tabla I).

Sobre la base de la información cariotípica de las especies chilenas de Alstroemeria publicada hasta la fecha (Buitendijk \& Ramanna 1996, Jara-Seguel et al. 2004 Baeza et al. 2007, 2008, 2010b), las especies de este género que presentan los valores más bajos de LTC $(\mu \mathrm{m})$ son $A$. graminea Phil., A. diluta Ehr.Bayer subsp. chrysantha Ehr.Bayer y $A$. versicolor (Tabla I). Estos valores no sobrepasan los $130 \mu \mathrm{m}$ y son los pares de cromosomas 1 , 2 y 3 los que representan los valores más altos de longitud relativa (sobre el 60\%). Otra característica interesante es la presencia siempre de un microsatélite en el brazo corto del cromosoma 3 en estas especies. En especies como $A$. cummingiana Herbert, A. hookeri Lodd. subsp. hookeri, A. 
aurea Graham, A. presliana Herbert, A. ligtu L., A. peregrina L. el valor de LTC sobrepasa los $180 \mu \mathrm{m}$ y los tres primeros pares de cromosomas constituyen menos del 30\% del LTC total (Baeza et al. 2007, 2010 b). Esto significa que el resto de los cromosomas en estas especies también contribuyen notoriamente en la longitud cromosómica relativa total de cada especie. Por otro lado, Alstroemeria graminea presenta el cariotipo más asimétrico y con el menor valor de LTC conocido hasta ahora entre las especies de Alstroemeria (LTC $=107,8 \mu \mathrm{m}$; AsK \%= 80,1\%; Syi= 24,8\%; Jara-Seguel et al. 2004), seguido por $A$. diluta subsp. chrysantha y $A$. versicolor (Tabla I). Sin embargo, A. graminea es la única especie anual dentro de Alstroemeria, con flores solitarias y sin protandría, caracteres considerados por Bayer (1998) como suficientes para crear un nuevo género endémico de Chile, Taltalia, con una sola especie, T. graminea Bayer. Actualmente, autores como Sanso \& Xifreda (2001) y Aagesen \& Sanso (2003) señalan que Taltalia graminea se trata de una especie de Alstroemeria. Aunque aun existen numerosas especies de Alstroemeria no estudiadas citológicamente, es probable que $A$. graminea continúe siendo la especie dentro del género con menor LTC y con los índices de asimetría del cariotipo más altos. En este contexto, $A$. versicolor y $A$. diluta ssp. chrysantha estarían bastante relacionadas con $A$. graminea por compartir características cariotípicas en términos de asimetría y valores de LTC.
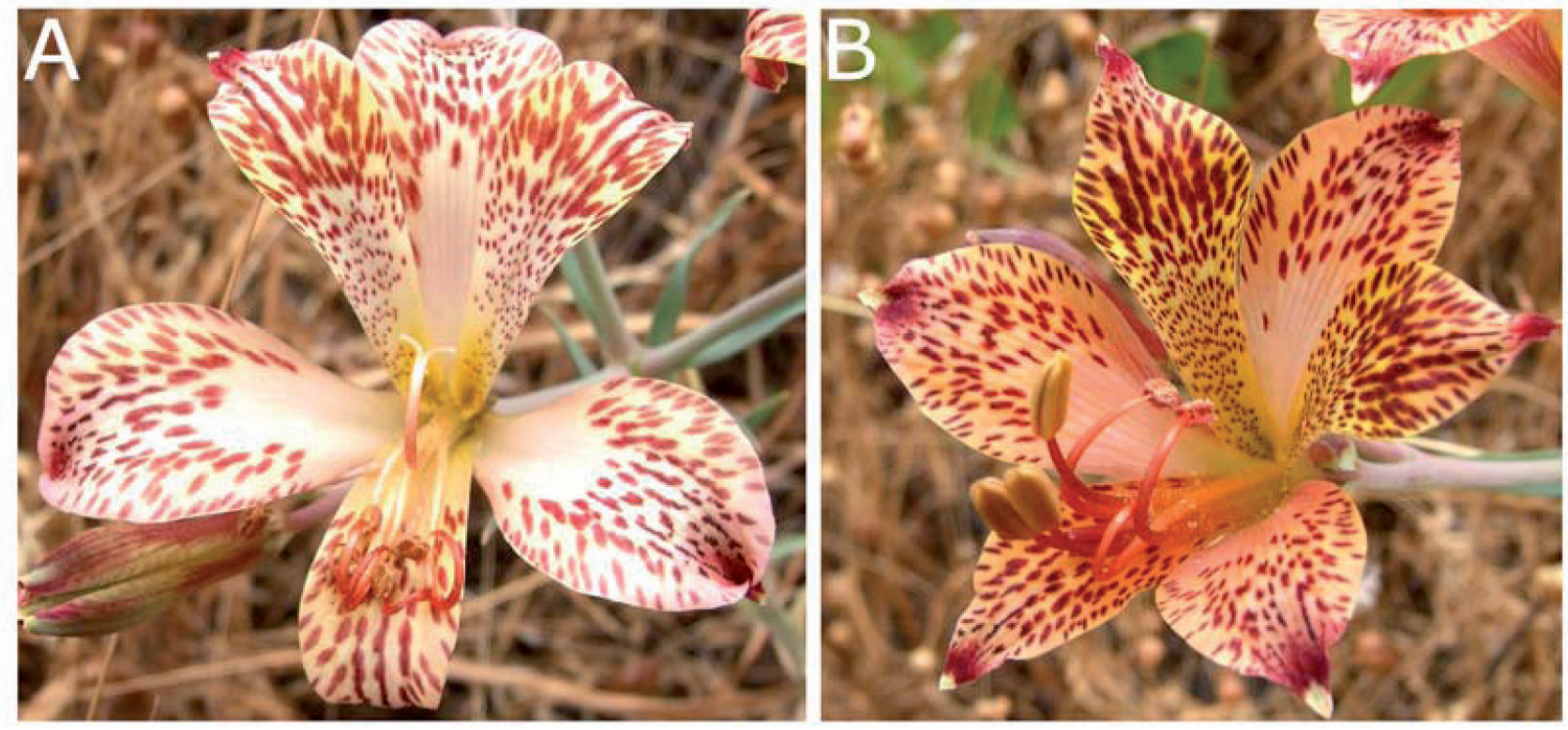

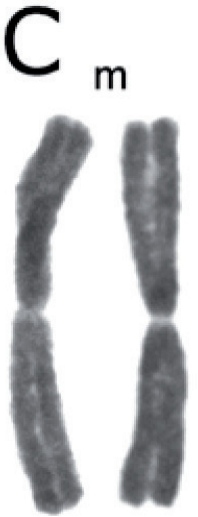

1 $\mathrm{sm}$

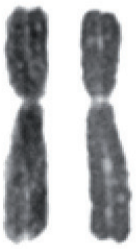

2 st

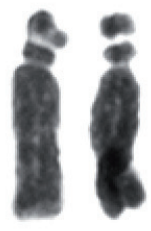

3

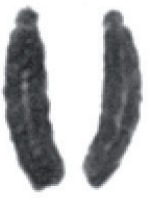

4 st

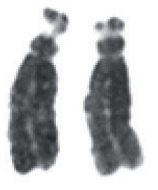

5
St
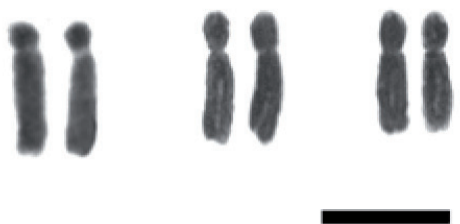

6 $\mathrm{sm}$

$\mathrm{sm}$

Figura 1. A-B: Fotografías de Alstroemeria versicolor C: Cariotipo de Alstroemeria versicolor. Escala $=5 \mu \mathrm{m}$.

Figure 1. A-B: Photographies of Alstroemeria versicolor C: Karyotype of Alstroemeria versicolor. Scale $=5 \mu \mathrm{m}$. 
TABla I. Comparación de las características del cariotipo de Alstroemeria diluta subsp. chrysantha, A. graminea y A. versicolor. LTC: longitud total de los cromosomas $(\mu \mathrm{m}) ;$ AsK \% = índice de asimetría de Arano y Saito (1980), Syi = índice de asimetría de Venora et al. (2002).

TABLE I. Comparison of karyotype characteristics of Alstroemeria diluta subsp. chrysantha, A. graminea and A. versicolor. LTC: total chromosome length $(\mu \mathrm{m})$; AsK \% = Asymmetry index of Arano and Saito (1980), Syi = Asymmetry index of Venora et al. (2002).

\begin{tabular}{|c|c|c|c|c|c|c|c|}
\hline & $\begin{array}{l}\text { LTC } \\
(\mu \mathrm{m})\end{array}$ & AsK \% & Syi & $\begin{array}{c}1+2+3 \\
(\mu \mathrm{m})\end{array}$ & cromosoma 1 & cromosoma 2 & cromosoma 3 \\
\hline A. diluta ssp. chrysantha & 119,6 & 71,4 & 40,0 & 62,6 & $\mathrm{~m}$ & $\mathrm{t}$ & t-sat \\
\hline A. graminea & 107,8 & 80,1 & 24,8 & 63,0 & $\mathrm{~m}$ & $\mathrm{sm}$ & st-sat \\
\hline A. versicolor & 129,0 & 65,9 & 51,8 & 68,0 & $\mathrm{~m}$ & $\mathrm{sm}$ & st-sat \\
\hline
\end{tabular}

\section{AGRADECIMIENTOS}

Se agradece al Departamento de Botánica y a la Dirección de Investigación de la Universidad de Concepción por las facilidades otorgadas.

\section{BIBLIOGRAFÍA}

Aagesen, L. \& M. SAnso. 2003. The phylogeny of the Alstroemeriaceae, based on morphology, rps16 Intron, and rbcL sequence data. Systematic Botany 28: 47-69.

Arano, H. \& H. Saito. 1980. Cytological studies in family Umbelliferae 5. Karyotypes of seven species in subtribe Seselinae. La Kromosomo 2: 471-480.

Arroyo, M. 1995. Plantas, hongos y líquenes. En: J. Simonetti, M. Arroyo, A. Spotorno \& E. Lozada (eds.), Diversidad biológica de Chile. Conicyt, Santiago, Chile: 5-7 pp.

Baeza, C., O. Schrader \& H. Budahn 2007. Characterization of geographically isolated accessions in five Alstroemeria L. species (Chile) using FISH of tandemly repeated DNA sequences and RAPD analysis. Plant Systematics and Evolution 269: 1-14.

Baeza, C., O. Schrader, E. Ruiz \& M. Negritto. 2008. Alstroemeria presliana Herb. (Alstroemeriaceae) in Chile from a cytogenetic perspective. Chilean Journal of Agricultural Research 68(4): 328-333.

BaEza, C., E. Ruiz\& P. NovoA. 2010a. The karyotype of Alstroemeria diluta Ehr. Bayer subsp. chrysantha (Alstroemeriaceae). Chilean Journal of Agricultural Research 70(4): 667-669.

Baeza, C., E. Ruiz \& M. Negritto. 2010b. Comparative karyotypic analysis in the Alstroemeria hookeri Lodd. (Alstroemeriaceae) complex sensu Bayer (1987). Genetics and Molecular Biology 33(1): 119-124.

BAYER, E. 1987. Die Gattung Alstroemeria in Chile. Mitteilungen der Botanischen Staatssamlungen München 24: 1-362.

BAYER, E. 1998. Taltalia, eine neue Gattung in der Familie Alstroemeriaceae. Sendtnera 5: 5-14.

ButtendiJ,, J., \& M. Ramanna. 1996. Giemsa C-banded karyotypes of eight species of Alstroemeria L. and some of their hybrids. Annals of Botany 78: 449-457.

Jara-Seguel, P., C. Palma-Rojas \& E. Brand. 2004. Karyotype and C-bands in the annual inca lily Alstroemeria graminea. Belgian Journal of Botany 137 (2): 199-204.

Levan, A, K. Fredga \& A. SAndberg. 1964. Nomenclature for centromeric position on chromosomes. Hereditas 52: 201220.

Muñoz, M. \& A. Moreira. 2003. Alstroemerias de Chile. Diversidad, distribución y conservación. Taller La Era, Santiago. 140 pp.

Sanso, M. \& C. Xifreda. 2001. Generic delimitation between Alstroemeria and Bomarea (Alstroemeriaceae). Annals of Botany 88: 1057-1069.

Venora, G., S. Blangiforti, M. Ruffini Castiglioni, D. Pignone, F. Losavio \& R. CRemonini. 2002. Chromatin organisation and computer arded karyotyping of Triticum durum Desf. cv Timilia. Caryologia 55: 91-98. 